\title{
GLOBULAR CLUSTER SYSTEMS OF ELLIPTICAL GALAXIES
}

\author{
STEPHEN E. ZEPF \\ Dept. of Astronomy, Yale University, New Haven, CT 06520 \\ AND \\ KEITH M. ASHMAN \\ Depts. of Physics, Univ. of Kansas, Lawrence, KS, 66045 \\ and Baker University, Baldwin, KS 66006
}

\begin{abstract}
.
We review the observed properties of globular cluster systems and their implications for models of galaxy formation. Observations show that globular clusters form in gas-rich mergers, and that bimodal metallicity distributions are common in the globular cluster systems of ellipticals, with the metal-poor population more extended than the metal-rich one. These are three of the four predictions of the simple merger model of Ashman \& Zepf (1992). The fourth prediction concerns the properties of the globular cluster systems of spirals, and is still to be tested by observation. Adopting Occam's razor, the confirmation of the fundamental predictions of the merger model from both young and old globular cluster systems is strong evidence that typical elliptical galaxies formed from the mergers of spiral galaxies. However, the simplifying assumptions of the Ashman-Zepf merger model limit its applicability to certain complex situations such as the formation of $\mathrm{cD}$ galaxies. We conclude this review by introducing new observational and theoretical programs that will further the understanding of the physical mechanisms of globular cluster and galaxy formation.
\end{abstract}

\section{Introduction}

The dramatic revolution in the understanding of globular cluster systems and their implications for galaxy formation can be traced by following the role the subject has played at major international meetings on mergers of galaxies. At the Heidelberg meeting in 1989, globular cluster systems (GCSs) were the focus of the "appointed skeptic" (van den Bergh 1990), whereas in Kyoto in 1997, globular clusters appeared prominently in the 
introductory talk as some of the strongest evidence that now quiescent elliptical galaxies formed from the past mergers of spiral galaxies (Schweizer 1998). This review will discuss the theoretical predictions and observational evidence that led to this revolution. We will then point to new directions that promise further advances in our understanding of globular cluster systems and their implications for the formation history of their host galaxy.

\section{What the Merger Model Predicted}

Ashman and Zepf (1992; hereafter AZ) carried out a detailed study of the relationship between galaxy mergers and globular cluster systems. If elliptical galaxies are the products of spiral galaxy mergers, globular clusters must form in such mergers (Schweizer 1987). This is because elliptical galaxies have a higher specific frequency (number per unit luminosity) of globular clusters than do spirals (van den Bergh 1990). By considering globular cluster formation efficiency and the gas content of the progenitor spiral galaxies, AZ showed that a sufficient number of globular clusters could form in major mergers to explain the specific frequency of globular clusters systems around ellipticals. This led to the conceptually simple prediction that, if elliptical galaxies are formed in galaxy mergers, ongoing galaxy mergers contain young globular clusters. In addition, AZ described the expected properties of young globular clusters, such as their blue colors, bright luminosities, and compact sizes. They also pointed out that HST observations of ongoing mergers like NGC 7252 and NGC 1275 should clearly reveal such objects. Thus the presence of young globular clusters in ongoing mergers constitutes a testable prediction that can be used to refute the merger model.

Perhaps the most unique prediction of the Ashman-Zepf model was that the globular cluster systems of elliptical galaxies formed in mergers have bimodal metallicity distributions. The progenitor spirals are expected to have a halo of metal-poor clusters, like those observed in the Milky Way and M31. More generally, in the context of the simple merger picture one expects such globular clusters to have formed out of gas that has experienced little metal enrichment. In contrast, globular clusters that form in the merger itself are expected to form out of the relatively enriched gas in the spiral disks. Thus these "merger-produced" globular clusters will have significantly higher metallicities than their counterparts in the halos of the progenitor spirals. For elliptical galaxies with "normal" specific frequencies (roughly double those of spirals), AZ showed that the number of metal-rich globular clusters formed in the merger must be comparable to the number of metal-poor globular clusters contributed by the progenitor spirals. Thus the overall metallicity distribution of the globular cluster systems of ellipticals is predicted to be bimodal, with roughly equal numbers of clusters in 
each "peak" of the distribution.

The third prediction of the Ashman-Zepf model is that, in an elliptical galaxy, the metal-rich cluster system will be more centrally concentrated than the metal-poor cluster system. This difference mirrors the different histories of the gas out of which the two globular cluster subsystems form. Compared to the low-metallicity halo gas that produces the metal-poor globular clusters, the enriched gas responsible for the metal-rich clusters is likely to have undergone more dissipation. This may arise as gas collapses from the halo to the disk in the progenitor spirals, or during the merger process itself. Consequently, the metal-rich cluster system is predicted to be more spatially concentrated than the metal-poor system. The effect may be increased by the tendency of mergers to "puff up" the stellar components of the progenitor galaxies. This would affect the metal-poor globular clusters, but not the dissipative gas out of which the metal-rich clusters form.

\section{What the Observations Showed}

Observational studies of globular cluster systems have advanced rapidly, so the fundamental predictions of the Ashman-Zepf model can now be tested. This section is devoted to a review of the relevant observations and the comparison of these to the model predictions.

\subsection{YOUNG GLOBULAR CLUSTERS}

The prediction that globular clusters form in gas-rich mergers has now been repeatedly confirmed, as reviewed in many places (e.g. Whitmore, these proceedings, Schweizer 1997, Ashman \& Zepf 1998). Specifically, HST observations of gas-rich mergers have uncovered a wealth of objects with bright luminosities, blue colors, and compact sizes, as predicted by AZ. These properties are all consistent with those expected of young objects with masses and sizes of the globular clusters in the Galaxy. Moderate resolution spectroscopy has further confirmed that these objects are composed of a young stellar population consistent with those given by standard stellar population models. This agreement includes the strength of the Balmer lines, when compared to LMC clusters (Bica \& Alloin 1986) or to updated stellar populations models and their improved stellar libraries are used, which were not available when the first spectra were published (e.g. Schweizer \& Seitzer 1993, Zepf et al. 1995a). Finally, high resolution spectroscopy of a few of the most nearby examples provides velocity dispersions indicative of masses that are typical of Galactic globular clusters, and agree well with those calculated from the observed colors and luminosities, combined with stellar population models (Ho \& Fillipenko 1997).

The prediction that globular clusters are formed in merger-induced starbursts is therefore confirmed. However, it is also important to recognize that 
well established physical processes will act to destroy some fraction of the initial young globular clusters (Fall \& Rees 1977 and subsequent papers). Such destruction is also suggested by the higher ratio of light in clusters to total light seen in galaxy mergers compared to any old system, even the halo of the Galaxy or cD galaxies (Zepf et al. 1998). Moreover, the net effect of these processes is to preferentially destroy low mass clusters, so it is possible to begin with a power-law mass function, and end-up with a lognormal mass function, like that observed in the galaxy (e.g. Gnedin \& Ostriker 1997).

\subsection{BIMODAL COLOR DISTRIBUTIONS}

The second prediction of the AZ merger model is that the globular cluster systems (GCSs) of elliptical galaxies will be composed of metal-poor populations from the progenitor spirals and metal-rich populations formed during the merger(s) that formed the elliptical. As described by Zepf \& Ashman (1993) and Ashman \& Zepf (1998), the metal-rich population will typically be significantly redder than the metal-poor population because most mergers occur at moderate or high redshift, and therefore metallicity differences dominate the broad-band colors.

Bimodality in the color distribution of the GGCs of elliptical galaxies was discovered by Zepf \& Ashman (1993). Using the best available data and mixture-modeling algorithms, they showed that the color distributions of NGC 4472 and NGC 5128 were likely to be bimodal. Data of much higher quality for a number of elliptical galaxies have since become available. This large body of evidence indicates that bimodality is the norm for bright ellipticals (e.g. Ashman \& Zepf 1998). Two typical examples are shown in Figure 1. In these and many other cases, mixture-modeling algorithms confirm objectively the significance of the bimodality apparent to the eye. The reader is referred to the Ashman \& Zepf (1998) book for plots of many more examples of color distributions of GCSs.

\subsection{COLOR GRADIENTS}

The third prediction of the AZ model is that the the metal-rich population is more spatially concentrated than the metal-poor population. Color gradients, which were suspected at the time of AZ and have now been confirmed (Ashman \& Zepf 1998 and references therein), are a natural result of the AZ model, although not unique to it. The key to testing the merger prediction is to compare directly the distributions of the blue and red populations around elliptical galaxies. This was first achieved by the Geisler et al. (1996) study of the NGC 4472 system. They showed that the metal-rich population is more spatially concentrated than the metal-poor population, thereby confirming the third prediction of the AZ merger model. 


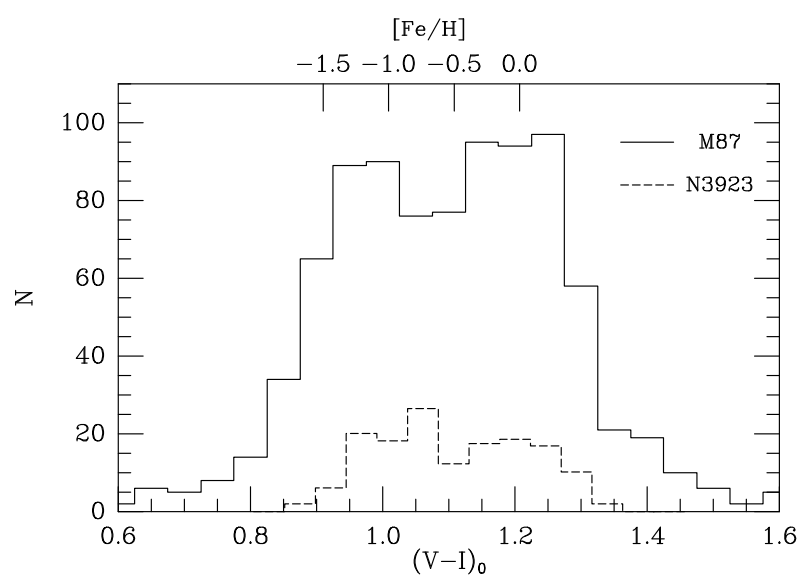

Figure 1. Color distributions for the GCSs of M87 from Whitmore et al. (1995) and NGC 3923 from Zepf et al. (1995b). The color distributions of both GCSs are bimodal, although the number of clusters observed in M87 is much greater

\subsection{OTHER CONSIDERATIONS}

While the fundamental predictions of the Ashman-Zepf model have been confirmed, there are specific cases where the quantitative agreement between prediction and observation breaks down. This is currently most clear for the number and precise color of the metal-poor population in elliptical galaxies (e.g. Zepf et al. 1995b, Forbes et al. 1997). In the simplest merger picture, the color and specific frequency $\left(S_{N}\right)$ of the blue population is constant because all progenitor spirals are assumed to have the same halo population of metal-poor clusters. Higher specific frequencies of clusters in some ellipticals are therefore attributed to increased formation (or survival) efficiency of metal-rich clusters created in the mergers that made these galaxies. However, the metal-poor globular cluster populations of ellipticals do not appear to have identical metallicities, and some high $S_{N}$ systems have an enhanced frequency of metal-poor clusters. Perhaps the clearest case is M87, for which the specific frequency of metal-poor clusters is about seven: much higher than the observed specific frequency of metal-poor globulars in spirals. However, this may not be universal, as the high $S_{N}$ of NGC 3311 appears to be due solely to metal-rich clusters (Secker et al. 1995).

It is clear that these observations indicate the breakdown of the simplifying assumptions of the model. Brighter ellipticals are unlikely to be the result of the merger of only two spirals; they are too massive. Further, progenitor spirals will not have identical cluster populations before the merger. Finally, accretion of smaller satellites is likely to play a role as well, as seems 
to be the case for the halo population of the Galaxy. The question is whether a more sophisticated and realistic treatment of the merger process is likely to preserve these two fundamental predictions of the Ashman-Zepf model. While a complete answer to this question requires detailed modeling (see Section 4), it seems inevitable that metallicity bimodality and the spatial concentration dichotomy will be preserved, at least in some ellipticals. This is because the merger model requires that major spiral-plus-spiral mergers are involved at some point in the formation of history of an elliptical. The number of such mergers and the likelihood that later mergers may be predominantly stellar are both unimportant, since typically globular clusters in the progenitor spiral halos will be metal-poor, and those formed in any gas-rich merger will be relatively metal-rich and spatially concentrated.

\section{New Observational and Theoretical Paths}

\subsection{THEORY}

One way to advance the model beyond the formation of an elliptical from the merger of two spirals is to place the model in a more specific cosmological context. In this way, the full merging history of elliptical galaxies can be followed statistically for a variety of possible models. Semi-analytic models of galaxy formation and evolution (e.g. Kauffmann et al. 1993, Cole et al. 1994) are well-suited for this program, and one of us (SEZ) is working with G. Kauffmann to implement this approach. A second area ripe for advancement is the theoretical understanding of the formation of globular clusters, as the wealth of new observational evidence in nearby merging galaxies has not yet been turned in to advances in the understanding of the formation of dense, bound stellar systems like globular clusters.

\subsection{OBSERVATION}

The kinematics of globular cluster systems provides valuable information about the formation history and mass distribution of the host galaxy. Until recently, it had proven to be difficult to obtain more than a few tens of clusters around a given galaxy. However, the increase in efficiency and areal coverage of multi-object spectrographs, as well as higher quality imaging for object selection, has opened up this field, even with 4-m class telescopes.

One of the best studied cases is M87 (Cohen \& Ryzhov 1997 and references therein). Here, we will focus on our work with R. Sharples and others on spectroscopy of globular clusters around NGC 4472 (Sharples et al. 1997). The most exciting result to come from our study is the tentative detection of kinematical differences between the metal-rich and metal-poor cluster populations in this galaxy. Specifically, the velocity dispersion of the

metal-rich clusters appears to be higher than that of the metal-poor clus- 

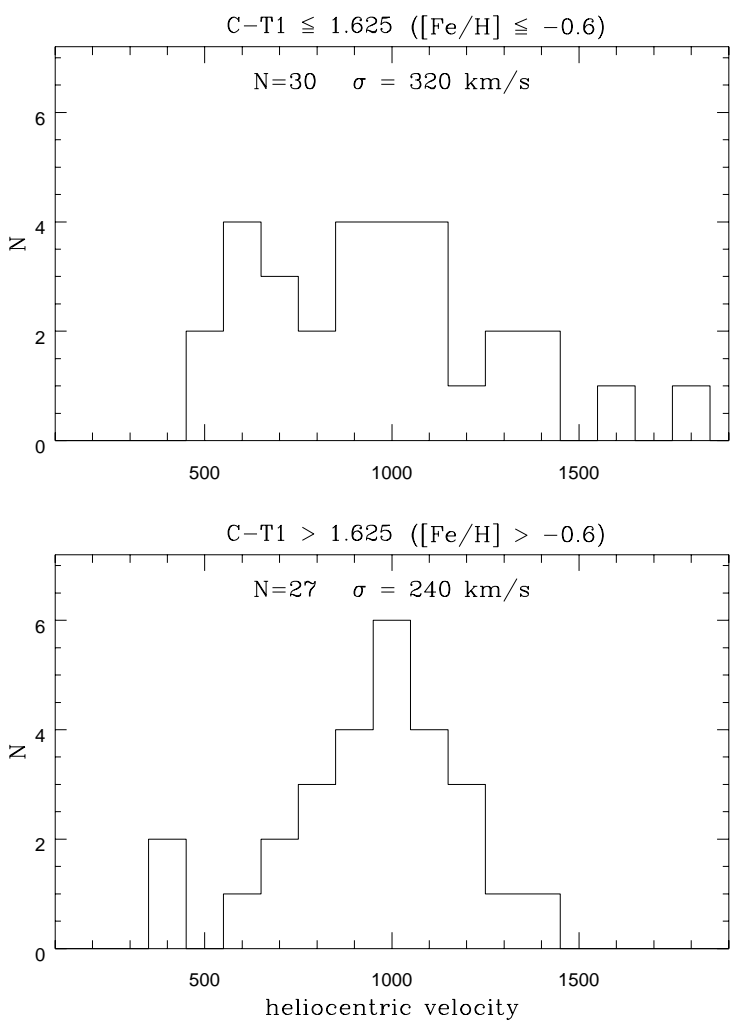

Figure 2. A comparison of the velocity dispersions of the metal-poor and metal-rich cluster populations in NGC 4472. An F-test rejects the hypothesis that these two have the same dispersion at the $86 \%$ confidence level.

ters (Figure 2). Moreover, the metal-poor clusters appear to rotate along the major axis, whereas the metal-rich clusters do not. At a basic level, the kinematics provide further physical evidence for the division of the cluster system into metal-poor and metal-rich subsystems that was originally based on an analysis of the colors alone. Furthermore, since the metal-rich cluster population is more spatially concentrated but has less rotation than the metal-poor cluster system, significant angular momentum transport must have occurred. This is seen in merger simulations, but is contrary to alternative models of episodic formation histories without mergers.

\section{Conclusions}

The merger model of Ashman \& Zepf (1992) made four predictions for properties of globular cluster systems that had not yet been tested by observation, and which were generally contrary to the standard picture at that time. Three of these four have now been confirmed by observation, while the fourth has yet to be tested. Although limitations of the original model have 
also been revealed by other observations, the striking agreement with many of the predictions suggests that the model is mostly correct. For it not to be correct, the young clusters observed in mergers would have to be irrelevant for globular cluster populations as a whole, even though their properties are exactly those expected for young globular clusters. Moreover, the bimodal metallicity distributions observed in elliptical galaxy GCSs would have to have formed in an episodic process other than mergers, that is also efficient at transporting angular momentum. Given these constraints, any model that successfully accounts for the observations is likely to be similar physically to the merger model. Globular clusters have greatly improved our understanding of the formation history of galaxies, and with further photometric observations and new spectroscopic data, they promise to continue to do so.

\section{Acknowledgements}

We thank our many collaborators on the various projects described above. Some of the research described above was supported by grants GO06092.01-94A, AR-06405.01-95A, and an award to SEZ from the Dudley Foundation. SEZ also acknowledges the support of a Hubble Fellowship during much of this work.

\section{References}

Ashman, K.M., \& Zepf, S.E. 1992, ApJ, 384, 50

Ashman, K.M., \& Zepf, S.E. 1998, Globular Cluster Systems (Cambridge: Cambridge University Press), in press

Bica, E., \& Alloin, D. 1986, A\&A, 162, 21

Cohen, J.G., \& Ryzhov, A. 1997, ApJ, 486, 230

Cole, S., Aragon-Salamanca, A., Frenk, C.S., Navarro, J., \& Zepf, S.E. 1994, MNRAS, 271,781

Forbes, D.A., Brodie, J.P., \& Grillmair, C.J. 1997, AJ, 113, 1652

Geisler, D., Lee., M.G., \& Kim, E. 1996, AJ, 111, 1529

Gnedin, O.Y., \& Ostriker, J.P. 1997, ApJ, 474, 223

Kauffmann, G., White, S.D.M., \& Guiderdoni, B. 1993, MNRAS, 264, 201

Ho, L.C. \& Filippenko, A.V. 1997, ApJ, 466, L83

Schweizer, F. 1987, in Nearly Normal Galaxies, ed. S.M. Faber (New York: Springer), 18

Schweizer, F. 1997, in The Nature of Elliptical Galaxies, eds. M. Arnaboldi, G.S. Da Costa, \& P. Saha (San Francisco: ASP), 447

Schweizer, F. \& Seitzer, P., 1993, ApJ, 417, L29

Sharples, R.M., Zepf, S.E., Bridges, T.J, et al. 1997, MNRAS, submitted

Secker, J., Geisler, D., McLaughlin, D., \& Harris, W.E. 1995, AJ, 109, 1019

van den Bergh, S. 1990, in Dynamics and Interactions of Galaxies, ed. R. Wielen (Berlin: Springer), 492

Whitmore, B.C. 1998, these proceedings

Whitmore, B.C., et al. 1995, ApJ, 454, L73

Zepf, S.E., \& Ashman, K.M. 1993 MNRAS, 264, 611

Zepf, S.E., Carter, D., Sharples, R.M. \& Ashman, K.M. 1995a, ApJ, 445, L19

Zepf, S.E., Ashman, K.M., \& Geisler, D. 1995b, ApJ, 443, 570 